\title{
ON A METHOD OF COMPARISON FOR TRIPLE-SYSTEMS*
}

\author{
BY \\ LOUISE D. CUMMINGS \\ INTRODUCTION
}

1. If $n$ elements $1,2,3, \cdots n$ can be distributed in triads in such a way that every pair of elements appears in one and only one triad, the totality of triads forms a triple-system of $n$ elements. Reiss $\dagger$ has shown that it is possible to form a triple-system of $n$ elements provided $n$ is of the form $6 m+1$ or $6 m+3$.

Different methods for constructing triple-systems have been given by Reiss, $\nmid$ Netto, $\ddagger$ Heffter, $\S$ and E. H. Moore;|| but methods for testing the non-congruency of these systems when formed are lacking. The group of. substitutions that transform a triple-system into itself has hitherto been adopted as the abstract mark of the system, and Zulauf $\llbracket$ has shown, by a consideration of the groups, that the four systems on 13 elements of Kirkman, Reiss, De Vries, and Netto are reducible to two incongruent systems whose groups are different. In the present paper it is shown however that for $n=15$ two incongruent triple-systems, $\Delta_{15}$, may have the same group.

By a simple process non-congruent triple-systems are constructed. These are used to illustrate a new method of comparison, by means of a new sort of abstract marks. This method requires no knowledge of the group but incidentally facilitates its determination. No exhaustive determination of every $\Delta_{15}$ which may be obtained by this process is here undertaken, but the 24 systems discussed include 12 apparently not hitherto constructed.

\section{Application of the method to a Reiss triple-system on 15 elements}

2. A triple-system formed by the Reiss method ${ }^{* *}$ is shown in the accompanying table, which gives 28 triples of the system; the remaining 7 , formed

\footnotetext{
* Presented to the Society, April, 1914.

† Ueber eine Steinersche kombinatorische Aufgabe, Crell e's J o u rn a l, vol. 56 (1859), pp. 326-344.

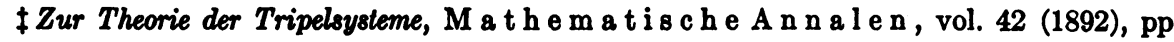
143-152.

8Ueber Tripelsysteme, Mat h e m a t is c h e An nale n, vol. 49 (1897), pp. 101-112. 285.

II Concerning Triple Systems, M a t h e mat i a c h e A n n a l e n, vol. 43 (1893), pp. 271-

T Ueber Tripelsysteme von 19 Elementen. Dissertation. Marburg, 1897.

** M. Reiss, l. c.
} 
from the elements which head the columns, are $a b d, a c g, a e f, b c e, b f g, c d f, d e g$.

TABLE I

\begin{tabular}{|c|c|c|c|c|c|c|}
\hline$a$ & $b$ & $c$ & $d$ & $e$ & $f$ & $g$ \\
\hline 87 & 86 & 85 & 84 & 83 & 82 & 81 \\
62 & 71 & 76 & 75 & 74 & 73 & 72 \\
53 & 52 & 31 & 61 & 65 & 64 & 63 \\
41 & 43 & 42 & 32 & 21 & 51 & 54 \\
\hline
\end{tabular}

This system may be arranged in the following rectangular array. Each element heads one column; below it are placed the 7 pairs of elements that occur with it in triads of the system.

\begin{tabular}{lllllllllllllll}
$a$ & $b$ & $c$ & $d$ & $e$ & $f$ & $g$ & 1 & 2 & 3 & 4 & 5 & 6 & 7 & 8 \\
\hline 87 & 86 & 85 & 84 & 83 & 82 & 81 & $a 4$ & $a 6$ & $a 5$ & $a 1$ & $a 3$ & $a 2$ & $a 8$ & $a 7$ \\
62 & 71 & 76 & 75 & 74 & 73 & 72 & $b 7$ & $b 5$ & $b 4$ & $b 3$ & $b 2$ & $b 8$ & $b 1$ & $b 6$ \\
53 & 52 & 31 & 61 & 65 & 64 & 63 & $c 3$ & $c 4$ & $c 1$ & $c 2$ & $c 8$ & $c 7$ & $c 6$ & $c 5$ \\
41 & 43 & 42 & 32 & 21 & 51 & 54 & $d 6$ & $d 3$ & $d 2$ & $d 8$ & $d 7$ & $d 1$ & $d 5$ & $d 4$ \\
$b d$ & $a d$ & $a g$ & $a b$ & $a f$ & $a e$ & $a c$ & $e 2$ & $e 1$ & $e 8$ & $e 7$ & $e 6$ & $e 5$ & $e 4$ & $e 3$ \\
$c g$ & $c e$ & $b e$ & $c f$ & $b c$ & $b g$ & $b f$ & $f 5$ & $f 8$ & $f 7$ & $f 6$ & $f 1$ & $f 4$ & $f 3$ & $f 2$ \\
$e f$ & $f g$ & $d f$ & $e g$ & $d g$ & $c d$ & $d e$ & $g 8$ & $g 7$ & $g 6$ & $g 5$ & $g 4$ & $g 3$ & $g 2$ & $g 1$
\end{tabular}

To examine the relations existing among the elements of the system, consider any triple, as $a 87$ and the 21 pairs of elements which are united with the 3 elements of this triple.

\begin{tabular}{|c|c|c|}
\hline$a$ & 8 & 7 \\
\hline 87 & $a 7$ & $a 8$ \\
62 & $b 6$ & $b 1$ \\
53 & $c 5$ & $c 6$ \\
41 & $d 4$ & $d 5$ \\
$b d$ & $e 3$ & $e 4$ \\
$c g$ & $f 2$ & $f 3$ \\
$e f$ & $g 1$ & $g 2$ \\
\hline
\end{tabular}

Begin with a pair in the column headed $a$, for example 62, written thus: call 6 the first element and 2 the second element of this pair; proceed from one column to the next in cyclical order, selecting in successive columns the pair which contains the second element of the preceding pair; continue this process until the pair to be selected from the first column is the initial pair. For the triple a87 the pairs would then appear as follows: $62,2 f, f 3,35,5 c$, $c 6,62,2 f$, the pairs repeating after each of the three columns has been examined twice. The occurring pairs, the periodicity, and the fact that the period consists of two rounds of the three columns can be shown conveniently by the notation $62 f / 35 c / 6$. The bar between two elements indicates the completion of one round of the three columns. Such a succession of pairs, repeating after 2 rounds of the columns, forms a sequence of period 2 .

Since the elements in the pair 62 can be written either as 62 or 26 , examine next this same pair with the two elements written in reverse order. Beginning with the pair 26 the pairs are selected from the columns as follows: $26,6 b, b 1$; $14,4 d, d 5 ; 53,3 e, e 4 ; 41,1 g, g 2 ; 26$, showing that after 4 rounds of the columns, the first column is again reached with the initial pair, or in sequence notation $26 b / 14 d / 53 e / 41 g / 2$. 
Apply this process to the remaining pairs $53,41, b d, c g$, ef, of column $a$. The pair 53 appears in the sequence of period 4 and does not require further examination, since 53 as initial pair would furnish the sequence $53 e / 41 \mathrm{~g} / 26 \mathrm{~b}$ $/ 14 d / 5$, which is identical with the above sequence of period 4 except as to starting point; hence any pair which has appeared in a sequence can be omitted from further examination. The pair 41 appears in the sequence of period 4; the pair $b d$ furnishes the sequence $b d 4 / e f 2 / g c 5 / d b 6 / c g 1 / b$, of period 5 ; the pair $f e$ leads to the sequence $f e 3 / f$ of period 1 ; the pair 87 to the sequence $87 a / 8$. The pair 78 leads from the body of the first column up to the element 8 at the head of the second column and the process cannot be continued. Therefore in examining the pairs of a column we omit the pair which, with the element at the head of the column, completes the triple under examination.

For the triple a87 the sequences are $62 f / 35 c / 6,26 b / 14 d / 53 e / 41 g / 2$, $b d 4 / e f 2 / g c 5 / d b 6 / c g 1 / b, f e 3 / f$; and all possible pairs for the column $a$, namely $62,26,53,35,41,14, b d, d b, c g, g c, e f, f e$, appear in these sequences. The number of rounds of the columns required to close a sequence (the period) is recorded for each sequence belonging to the triple under examination, and we shall call these numbers the index of that triple, and the longest sequence the major sequence.

The index of the triple a87 consists of the numbers $2,4,5,1$. The index shows that the sequences for the triple $a 87$ consist of a sequence closed in two cycles of three elements, hence containing two triads, a second sequence containing four triads, a third sequence containing five triads and a fourth sequence containing one triad. In any column twelve pairs must be considered and each pair occurs in one and only one round of the columns and therefore in one and only one triad in the sequences. Hence for a triple-system on 15 elements, the sum of the periods for every index is 12 . Indices of the types 111117, 111144,66 are written in the abbreviated form $1^{5} 7,1^{4} 4^{2}, 6^{2}$.

The sequences are closed cycles, in which any element may be selected as initial element and the sequence may be read in direct or reverse order. The sequences for $a 87,87 a$, and $8 a 7$ illustrate the following

THEOREM. The index of every triple in a given triple-system is invariant under all permutations of its triad.

\section{ANALYSIS OF THE MAJOR SEQUENCES}

3. An element which appears once, twice, three times in a sequence is designated as a single, double, triple point, respectively, for that sequence. It is evident that a sequence of period 1 contains no double point and hence that no duplicate element occurs in a triad of a sequence. 
LEMMA. A sequence of period greater than one contains no repeition in any quintad of elements.

\begin{tabular}{|c|c|c|}
$x$ & $y$ & $z$ \\
\hline$a b$ & $b c$ & $c d$ \\
$d e$ & &
\end{tabular}

Let the sequence for $x y z$ begin in the column $x$ with the initial pair $a b$, and for convenience number the places in the sequence. The elements in places 1,2 , and 3 may be $a, b$, and $c$, but the element in the place 4 is not $a$, since that would close the sequence. No repetition occurs in a triad of a sequence, hence the element in place 4 is neither $b$ nor $c$, and therefore must be a new element $d$. The element to fill place 5 forms with $d$ a pair in column $x$ and so is neither $a$ nor $b$; since this required element forms a triad with $c, d$, it must be a new element $e$. Hence no repetition occurs in a quintad of elements. By applying the above Lemma and reading the sequence forward and backward we obtain the following

THEOREM. A sequence of period equal to or less than three contains no multiple point.

4. 'This process applied to the 35 triples of the Reiss $\Delta_{15}$ gives indices and sequences only of certain types, namely:

TrIPLE

$a b d$

(3)

$a 87$

$a 41$
INDEX

$1^{5} 7$

1245

$6^{2}$

\section{SEquences}

$871 / 625 / 786 / 143 / 268 / 417 / 534 / 8$, $352 / 3, \quad c g f / c, \quad g c e / g, \quad e f g / e, \quad f e c / f$; $62 f / 35 c / 6,26 b / 14 d / 53 e / 41 g / 2$, $b d 4 / e f 2 / g c 5 / d b 6 / c g 1 / b, f e 3 / f$; $87 e / 26 f / 53 b / 78 d / 62 c / 35 g / 8$, $b d 8 / g c 2 / e f 6 \prime^{\prime} d b 3 / c g 5 / f e 7 / b$.

The indices have separated the 35 triples of the Reiss system into 3 classes, (1), (2), and (3). This separation into classez will be found to simplify the problem of determining the group of the system. For every substitution which converts the system into itself must permute among themselves the triples of a class, since formation of cycles depends on identity or difference of elements in the different columns and these are not changed by any substitution.

The 35 triples arranged in classes according to their indices are shown in the following table.

Classification of the Reiss $\Delta_{16}$ by indices

\begin{tabular}{|c|c|c|c|c|c|c|c|c|c|c|c|}
\hline \multicolumn{2}{|r|}{$1^{6} 7$} & \multicolumn{7}{|c|}{1245} & \multicolumn{3}{|c|}{$\hat{6}^{2}$} \\
\hline $\begin{array}{l}a b d \\
a c g \\
a e f\end{array}$ & $\begin{array}{l}b c e \\
b f g \\
c d f\end{array}$ & $\begin{array}{l}a 26 \\
a 35 \\
a 78\end{array}$ & $\begin{array}{l}b 25 \\
b 34 \\
b 68\end{array}$ & $\begin{array}{l}c 24 \\
c 58 \\
c 67\end{array}$ & $\begin{array}{l}d 23 \\
d 48 \\
d .57\end{array}$ & $\begin{array}{l}e 38 \\
e 47 \\
e 56\end{array}$ & $\begin{array}{l}f 28 \\
f 37 \\
f 46\end{array}$ & $\begin{array}{l}g 27 \\
g 36 \\
g 45\end{array}$ & $\begin{array}{l}a 14 \\
b 17 \\
c 13\end{array}$ & $\begin{array}{l}d 16 \\
e 12 \\
f 15\end{array}$ & $g 18$ \\
\hline & (1) & \multicolumn{7}{|c|}{ (2) } & \multicolumn{3}{|c|}{ (3) } \\
\hline
\end{tabular}


In the major sequence $871 / 625 / 786 / 143 / 268 / 417 / 534 / 8$ of the triple $a b d$, the second 8 enters in the seventh place after the first 8 , the third 8 in the seventh place after the second 8 , and the first 8 in the seventh place after the third 8 ; we denote this by the symbol $8(7,7,7)$; with this notation the elements of the sequence are characterized as follows: $8(7,7,7), 7(5,11,5)$, $1(7,7,7), 6(5,5,11), 2(8,13), 5(13,8), 4(5,5,11), 3(8,13)$. For a sequence of period 7 containing 21 elements the symbols $(5,11,5)$, $(5,5,11),(11,5,5)$ are identical and $(8,13) \equiv(13,8)$. Hence this sequence contains two distinct types of triple points and one type of double points.

The analysis of the major sequences of the triples of class (1) is given in the following table:

$\begin{array}{ccccccccc}\text { Cusss }(1) & (7,7,7) & (5,11,5) & (7,7,7) & (5,5,11) & (8,13) & (13,8) & (5,5,11) & (8,13) \\ a b d & 8 & 7 & 1 & 6 & 2 & 5 & 4 & 3 \\ b c e & 4 & 3 & 1 & 2 & 5 & 8 & 7 & 6 \\ c d f & 7 & 6 & 1 & 5 & 8 & 4 & 3 & 2 \\ \text { deg } & 3 & 2 & 1 & 8 & 4 & 7 & 6 & 5 \\ e f a & 6 & 5 & 1 & 4 & 7 & 3 & 2 & 8 \\ \text { fgb } & \mathbf{2} & \mathbf{8} & 1 & 7 & 3 & 6 & 5 & 4 \\ \text { gac } & 5 & 4 & 1 & 3 & 6 & 2 & 8 & 7\end{array}$

Determination of the GROUP FOR the REISs $\Delta_{15}$

5. Any operation of the group that leaves a triple-system invariant can only transform any triad and its sequences into itself, or into another of the same class. Therefore to find the group, we examine first for substitutions that transform into itself one of the longest sequences, and secondly for those that transform it into the remaining sequences of its class. Substitutions when determined must be tested on the whole triple-system.

An examination of the table of indices for this system shows that the 15 elements do not enter symmetrically as members of the triads in the classes, for example, the 7 triads in class (1) contain only the elements $a, b, c, d, e, f, g$. Since only those elements may be permuted which occur the same number of times in a class, the enumeration of the appearances of each element in the classes, as in the following table, shows the possible sets of transitive elements.

\begin{tabular}{|l|lllllllllllllll|}
\hline & $a$ & $b$ & $c$ & $d$ & $e$ & $f$ & $g$ & 1 & 2 & 3 & 4 & 5 & 6 & 7 & 8 \\
\hline$(1)$ & 3 & 3 & 3 & 3 & 3 & 3 & 3 & & & & & & & & \\
$(2)$ & 3 & 3 & 3 & 3 & 3 & 3 & 3 & & 6 & 6 & 6 & 6 & 6 & 6 & 6 \\
$(3)$ & 1 & 1 & 1 & 1 & 1 & 1 & 1 & 7 & 1 & 1 & 1 & 1 & 1 & 1 & 1 \\
\hline
\end{tabular}

The systems of transitivity for the group are therefore $a, b, c, d, e, f, g ; 1$; $2,3,4,5,6,7,8$.

The substitutions may be determined from any class in the system, but most easily from the class containing the longest sequences since these exhibit 
more repetitions of the elements. In the present case class (1) is therefore selected.

(1) Examine for substitutions to transform the triple $a b d$ into itself. The major sequence of $a b d$ is 871625786143268417534 , and a consideration of the possible rotations of this sequence into itself will determine the substitutions which transform the triple abd into itself. The element 1 forms a transitive system; hence a rotation of period 3 given by (1) (647) (235) (8) transforms this sequence into itself. The triple-system will now enable us to complete the substitution, for if two elements in one triple transform into two elements in a second triple, the third element in the first triple transforms into the third element in the second triple, for example, $18 g$ transforms into 18g. An application of both cycles (235) and (647) to the two triples $26 a$ and $24 c$ gives respectively $26 a, 34 b, 57 d ; 24 c, 37 f, 56 e$ and shows for the required substitution additional cycles $(a b d)$ and $(c f e)$. Hence the substitution $(a b d)(c f e)(g)(1)(235)(476)(8)$ transforms abd and its sequences into itself.

Any rotation of period 2 which transforms the sequence of $a b d$ into itself must keep fixed one of the elements 1 and interchange elements at equal distances from this 1 , for example, (1) (46) (38) (27) $\cdots$, but in the sequence 3 is a double point and 8 is a triple point and it is not possible to convert the sequence into itself by the interchange of two points of different multiplicities; hence no substitution of period 2 exists which will convert this sequence into itself. Therefore only a sub-group of order 3 transforms the triple $a b d$ into itself.

(2) Examine for substitutions to transform the triple $a b d$ into the other triples in its class. The table of class (1) exhibits the cycles (8473625) (1). If now we apply these two cycles to any triple in the system, as $a 14$, then the triples of the system appear in the following succession: $14 a, 17 b, 13 c, 16 d$, $12 e, 15 f, 18 g$, showing the cycle ( $a b c d e f g$ ) and this completes the substitution $s_{2} \equiv(a b c d e f g)$ (1) (2584736) which transforms $a b d$ in succession into every other triple in its class. Therefore the group for the Reiss system is of order 21 and is generated by

$s_{1} \equiv(a b d)(c f e)(g)(1)(235)(476)(8), \quad s_{2} \equiv(a b c d e f g)(1)(2584736)$. The method of construction employed by Reiss involves a cyclic substitution of order 7 , hence the appearance of $s_{2}$ was to be expected; but it did not show the substitution of order 3 .

\section{CONSTRUCTION OF NEW SYSTEMS}

6. The seven triples containing only the elements which head the columns in the seven-by-four array, Table I, \$2, form a triple-system on 7 elements, which is designated as a head for this array. The seven triples in the head 
and the twenty-eight triples in the array make up the thirty-five triples in the system. New systems may be formed by retaining the twenty-eight triples of the seven-by-four array and changing the seven triples of the head or by changing the seven-by-four array. An index determined from a sevenby-four array is designated as a partial index to distinguish it from a complete index determined from a fifteen-by-seven array.

Formation OF NEW SYSTEMS BY CHANGING THE HEAD OF THE SEVEN-BY-FOUR ARRAY

7. From the seven elements $a, b, c, d, e, f, g$ thirty-five triples can be formed. We determine for each of these triples its partial index from the seven-by-four array I. For example:

\begin{tabular}{|c|c|c|c|c|c|c|c|c|c|c|c|}
\hline \multirow{3}{*}{$\begin{array}{c}\text { TRIPLE } \\
a b c \\
a b d\end{array}$} & \multirow{2}{*}{\multicolumn{3}{|c|}{$\begin{array}{c}\text { INDEX } \\
1^{2}, 2,4 \\
1,7\end{array}$}} & \multicolumn{8}{|c|}{ Sequences } \\
\hline & & & & \multirow{2}{*}{\multicolumn{7}{|c|}{$\begin{array}{l}786 / 7, \quad 871 / 352 / 417 / 625 / 8, \quad 268 / 534 / 2, \\
871 / 625 / 786 / 143 / 268 / 417 / 534 / 8, \quad 352 / 3\end{array}$}} & \multirow[t]{2}{*}{$143 / 1 ;$} \\
\hline & & & & & & & & & & & \\
\hline \multicolumn{7}{|c|}{$1^{2}, 2,4$} & \multicolumn{4}{|c|}{1,7} & \\
\hline $\begin{array}{l}a b c \\
a b e \\
a b g\end{array}$ & $\begin{array}{l}\text { ace } \\
\text { acf } \\
\text { ade }\end{array}$ & $\begin{array}{l}\text { adf } \\
\text { adg } \\
\text { afg }\end{array}$ & $\begin{array}{l}b c d \\
b c f \\
b d f\end{array}$ & $\begin{array}{l}\text { bdg } \\
\text { bef } \\
\text { beg }\end{array}$ & $\begin{array}{l}\text { cde } \\
\text { cdg } \\
\text { ceg }\end{array}$ & $\begin{array}{l}c f g \\
e f g \\
\text { def }\end{array}$ & $\begin{array}{l}a b d \\
a b f \\
a c d\end{array}$ & $\begin{array}{l}a c g \\
b c e \\
b c g\end{array}$ & $\begin{array}{l}\text { bde } \\
\text { cdf } \\
\text { cef }\end{array}$ & $\begin{array}{l}\text { aef } \\
a e g \\
b f g\end{array}$ & $\begin{array}{l}d e g \\
d f g\end{array}$ \\
\hline
\end{tabular}

Select any triple, as $a b d$. Construct all the triple-systems on the seven elements $a, b, c, d, e, f, g$, which contain $a b d$, and retain one of each type. Six systems $\Delta_{7}$ can be formed, but the sets of partial indices of four of those systems are identical. Hence this triple furnishes only three types of head for the seven-by-four array, namely $A, B$, and $C$, as in the table.

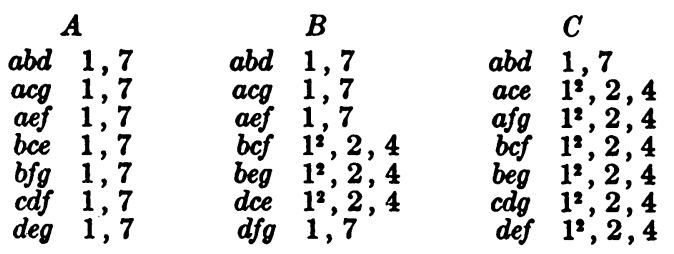

Since triples with different partial indices may give different types of heads, we examine in the same way the triple abe; this furnishes 4 heads of type $C$ and 2 heads of type $B$.

The 35 triples formed of the elements $a, b, c, d, e, f, g$ contain only 2 partial indices, $1^{2} 24$ and 17, and all triple-systems of seven elements have been formed for one triple selected from each class of partial indices, and three types of heads $A, B$, and $C$ have appeared. The twenty-eight triples in the seven-by-four array completed with any one of these heads form a triplesystem on 15 elements. 
The array, Table I, completed by these three heads, is denoted by $I A$, $\mathrm{I} B, \mathrm{I} C$; of these $\mathrm{I} A$ is the Reiss system already investigated.

\begin{tabular}{|c|ccccccc|}
\hline & $a$ & $b$ & $c$ & $d$ & $e$ & $f$ & $g$ \\
\hline & 87 & 86 & 85 & 84 & 83 & 82 & 81 \\
$I$ & 62 & 71 & 76 & 75 & 74 & 73 & 72 \\
& 53 & 52 & 31 & 61 & 65 & 04 & 63 \\
& 41 & 43 & 42 & 32 & 21 & 51 & 54 \\
\hline IA & $a b d$ & $a c g$ & $a e f$ & $b c e$ & $b f g$ & vilf & deg \\
IB & $a b d$ & $a c g$ & aef & $b c f$ & $b e g$ & $c d e$ & If \\
IC & abd & ace & afg & bcf & beg & cdg & def \\
\hline
\end{tabular}

To show that the 3 systems $\mathrm{I} A, \mathrm{I} B, \mathrm{I} C$, are non-congruent, it is necessary to write each system in the form of the fifteen-by-seven array and to obtain the indices for the 35 triples of each system; since it is possible that triples with different partial indices may have the same complete index and vice versa.

No two congruent systems have been admitted, but it does not follow that non-congruent systems have not been excluded, and in an exhaustive determination of all systems $\Delta_{15}$ every head must be applied to the seven-by-four array, and the indices for each system determined irom its fifteen-by-seven array.

8. Classification of IB by indices and transitive elements

\begin{tabular}{|c|c|c|c|c|c|c|c|c|c|c|c|}
\hline $1^{6} 24$ & $1^{5} 7$ & $1^{2} 234$ & $1^{2} 2^{3} 4$ & $1^{2} 46$ & \multicolumn{2}{|c|}{1245} & $2^{3} 6$ & \multicolumn{2}{|c|}{$2^{2} 4^{2}$} & $6^{3}$ \\
\hline$b c f$ & $a b d$ & $d 23$ & $b 25$ & $b 17$ & $a 87$ & $b 68$ & $f 28$ & $d 16$ & $b 34$ & $d 48$ & $a 14$ \\
$b e g$ & $a c g$ & $f 37$ & $e 67$ & $c 13$ & $a 26$ & $e 56$ & $g 36$ & $f 15$ & $c 24$ & $f 46$ & \\
$c d e$ & $a e f$ & $g 27$ & $e 38$ & $e 12$ & $a 35$ & $c 58$ & $d 57$ & $g 18$ & $e 47$ & $g 45$ & \\
\cline { 2 - 4 } & & & & & & & & & & & \\
\hline
\end{tabular}

(1)

(2)

(3)

(4)

(5)

(6)

(7)

(8)

The enumeration of the entrances of the elements in the 9 classes shows that for the group of the system the sets of transitive elements are $a ; b, c, e$; $d, f, g ; 1 ; 2,3,7 ; 5,6,8 ; 4$. 'These sets of transitive elements subdivide the classes into sets of triples which are not transformable into one another by operations of the group of the system; the subdivisions are shown by lines separating the triples in a class, and the system $I B$ contains 13 subdivisions. The group is generated by $s \equiv(a)(b e c)(d f g)(1)(237)(586)(4)$, and is of order 3 .

\section{Classification of IC by indices and transitive elements}

\begin{tabular}{|c|c|c|c|c|c|c|c|c|c|c|c|c|}
\hline \multicolumn{2}{|c|}{$1^{6} 24$} & \multirow{2}{*}{$\frac{1^{b} 7}{a b d}$} & \multirow{2}{*}{$\begin{array}{c}1^{3} 234 \\
c 58 \\
e 38 \\
f 28\end{array}$} & \multicolumn{2}{|c|}{$1^{2} 2^{3} 4$} & \multicolumn{2}{|c|}{$1^{2} 46$} & \multicolumn{2}{|c|}{1245} & \multirow{2}{*}{$\begin{array}{l}2^{2} 4^{2} \\
a 26 \\
b 34 \\
d 57\end{array}$} & \multirow{2}{*}{$\begin{array}{c}23^{2} 4 \\
c 67 \\
e 47 \\
f 46\end{array}$} & \multirow{2}{*}{$\frac{6^{2}}{g 18}$} \\
\hline $\begin{array}{l}\text { ace } \\
\text { def } \\
b c f\end{array}$ & $\begin{array}{l}\text { beg } \\
\text { cdg } \\
\text { afg }\end{array}$ & & & $\begin{array}{l}a 35 \\
b 25 \\
d 23\end{array}$ & $\begin{array}{l}g 27 \\
g 36 \\
g 45\end{array}$ & $\begin{array}{l}a 14 \\
b 17 \\
d 16\end{array}$ & $\begin{array}{l}c 13 \\
e 12 \\
f 15\end{array}$ & $\begin{array}{l}a 78 \\
b 68 \\
d 48\end{array}$ & $\begin{array}{l}c 24 \\
e 56 \\
f 37\end{array}$ & & & \\
\hline
\end{tabular}

(1)

(2) (3)

(4)

(5)

(6)

(7) (8)

(9) 
The sets of transitive elements for the system are $a, b, d ; c, e, f ; g ; 1 ; 2,3,5$; $4,6,7 ; 8$. These with the indices separate the system into 13 non-permutable subdivisions. The group is generated by $\& \equiv(a d b)(c e f)(g)(1)(253)$ (467) (8), and is of order 3.

10. A comparison of $I B$ and $I C$ shows that the groups of these two noncongruent systems are the same. For these groups are generated respectively by $s \equiv(a)(b e c)(d f g)(1)(237)(4)(586), t \equiv(a d b)(c e f)(g)(1)$ (253) (467) (8), and are abstractly the same, since each of these substitutions permutes 12 elements, in 4 cycles, each of order 3 , and leaves the remaining 3 elements fixed. But the system IB contains 4 triples with index $1^{5} 7$, while the system $I C$ contains only 1 triple with index $1^{5} 7$. Now in a given system the index of a triple is invariant under all the substitutions of the symmetric group; two systems are, therefore, certainly incongruent if their sets of indices are unlike, whether their groups are difierent or identical.* Hence no substitution exists which converts $\mathrm{I} B$ into $\mathrm{I} C$, and the two systems are non-congruent. That is, as pointed out in $\$ 1$, the same group belongs to two non-congruent systems.

Up to the present time the group has been considered as the abstract mark of a system. It is true that two systems with different groups are distinct, but as shown above, the converse is not true, a fact which apparently has not been suspected before.

FORMATION OF NEW SYSTEMS BY CHANGING THE FUNDAMENTAL SEVEN-BYFOUR ARRAY

11. If a seven-by ofour array shows 3 columns of the types $x, y$, and $z$ containing the 3 sets of pairs $12,34: 13,24$ : 14,23 which can be formcd from the four elements 1,2 , 3,4 , the four elmerts constitute a quadrangular array which may be denoted by the symbol [34]. The remaining

\begin{tabular}{|c|c|c|}
\hline$x$ & $y$ & $z$ \\
\hline 12 & 13 & 14 \\
34 & 24 & 23 \\
56 & 57 & 58 \\
78 & 68 & 67 \\
\hline
\end{tabular}
four elements $5,6,7,8$ must necessarily form also a quadrangular array; hence quadrangular arrays enter into a seven-by.four array always by couples, and it is easily shown that a, seven-by-four array must contain $0,2,6$, or 14 quadrangular arrays; - a fact hitherto not noticed, but of considerable importance in the following discussion.

An examination of every combination of two pairs occurring in the 7 columns of Table I shows that this seven-by-four array contains no quadrangular array.

Let us form, then, a new fundamental Table II, which shall contain two and only twe of these quadrangular arrays. In constructing columns suitable for a seven-byyfour array, the first pair in the column may be selected in 7 ways (since with the first element we may unite any one of the remaining

\footnotetext{
* The trutk of the converse is not here asserted, though it seems proibable.
} 
7 elements), the second pair in 5 ways, the third pair in 3 ways, and the fourth pair only in 1 way; hence 105 suitable columns may be formed.

From the 105 columns we select 3 columns $a, b, c$, containing the 2 quadrangular arrays $\left[\begin{array}{ll}1 & 2 \\ 3\end{array}\right]$ and $\left[\begin{array}{ll}5 & 6 \\ 7 & 8\end{array}\right]$. The remaining 4 columns $d, e, f, g$, must be selected so that no more quadrangular arrays are introduced, and the entering columns must not contain any pair that appears in the columns already in the table.

Table II

\begin{tabular}{|ccccccc|}
\hline$a$ & $b$ & $c$ & $d$ & $e$ & $f$ & $g$ \\
\hline 87 & 86 & 85 & 84 & 83 & 82 & 81 \\
65 & 75 & 76 & 73 & 72 & 71 & 74 \\
43 & 42 & 41 & 61 & 64 & 63 & 62 \\
21 & 31 & 23 & 25 & 15 & 45 & 53 \\
\hline
\end{tabular}

The Table II shows one of the several possible ways of constructing a sevenby-four array of the desired type. The determination, from the seven-byfour array II, of the partial indices for the 35 triples which may be formed from the 7 elements $a, b, c, d, e, f, g$, shows 5 different partial indices. The re-heading process is now applied to Table II. A triple of each index furnishes 6 systems $\Delta_{7}$. By the selection of 1 system of each type from these 30 , the 6 different heads $A, B, C, D, E, F$ shown in the following table are obtained for the seven-by-four array II.

\begin{tabular}{|c|c|c|c|c|c|c|c|c|c|c|c|c|}
\hline \multirow{3}{*}{ II } & $a$ & $b$ & $c$ & $d$ & $e$ & $f$ & $g$ & \multirow{2}{*}{\multicolumn{5}{|c|}{$\begin{array}{l}\text { NUMBER OF TRIPLES OF THE } \\
\text { HEAD WITH PARTIAL INDICES }\end{array}$}} \\
\hline & $\begin{array}{l}87 \\
65 \\
43\end{array}$ & $\begin{array}{l}86 \\
75 \\
42\end{array}$ & $\begin{array}{l}85 \\
76 \\
41\end{array}$ & $\begin{array}{l}84 \\
73 \\
61\end{array}$ & $\begin{array}{l}83 \\
72 \\
64\end{array}$ & $\begin{array}{l}82 \\
71 \\
63\end{array}$ & $\begin{array}{l}81 \\
74 \\
62\end{array}$ & & & & & \\
\hline & & 31 & & & & & 35 & $1^{8}$ & $1^{4} 2^{2}$ & $1^{2} 24$ & 24 & $4^{2}$ \\
\hline $\begin{array}{l}A \\
B \\
\boldsymbol{C} \\
D \\
\boldsymbol{E} \\
\boldsymbol{F}\end{array}$ & $\begin{array}{l}\text { bde } \\
\text { bde } \\
\text { bde } \\
\text { bde } \\
\text { bde } \\
\text { bde }\end{array}$ & $\begin{array}{l}a b c \\
a b f \\
a b f \\
a b g \\
b a c \\
a b g\end{array}$ & $\begin{array}{l}b f g \\
b c g \\
b c g \\
b c f \\
b f g \\
b c f\end{array}$ & $\begin{array}{l}\text { adf } \\
\text { acd } \\
\text { adg } \\
\text { acd } \\
\text { dag } \\
\text { adf }\end{array}$ & $\begin{array}{l}c d g \\
d f g \\
c d f \\
d f g \\
c d f \\
c d g\end{array}$ & $\begin{array}{l}\text { aeg } \\
\text { aeg } \\
\text { ace } \\
\text { aef } \\
\text { aef } \\
\text { ace }\end{array}$ & $\begin{array}{l}\text { cef } \\
\text { cef } \\
\text { efg } \\
\text { ceg } \\
\text { ceg } \\
\text { efg }\end{array}$ & $\begin{array}{l}1 \\
0 \\
0 \\
0 \\
1 \\
0\end{array}$ & $\begin{array}{l}\mathbf{0} \\
\mathbf{0} \\
\mathbf{2} \\
\mathbf{2} \\
\mathbf{4} \\
\mathbf{0}\end{array}$ & $\begin{array}{l}\mathbf{4} \\
2 \\
1 \\
1 \\
2 \\
2\end{array}$ & $\begin{array}{l}\mathbf{2} \\
\mathbf{2} \\
\mathbf{1} \\
\mathbf{2} \\
\mathbf{0} \\
\mathbf{3}\end{array}$ & $\begin{array}{l}\mathbf{0} \\
\mathbf{3} \\
\mathbf{3} \\
\mathbf{2} \\
\mathbf{0} \\
\mathbf{2}\end{array}$ \\
\hline
\end{tabular}

If a system $\Delta_{15}$ contains more than one $\Delta_{7}$, then for every such contained $\Delta_{7}$ there is an arrangement of the $\Delta_{15}$, showing this $\Delta_{7}$ as a head to a suitable seven-by-four array. These different arrangements may furnish dissimilar partial indices. For example, the system II $A$ contains three systems $\Delta_{\boldsymbol{7}}$ formed of the elements $a, b, c, d, e, f, g ; a, b, c, 1,2,3,4$; and $a, b, c$, $5,6,7,8$. The second of these heads, placed over the seven-by-four array formed of the 28 remaining triples of the system II $A$, gives for the triples $a b c, a 43, a 12, b 42, b 31, c 41, c 23$, respectively, the following partial indices $1^{8}, 1^{4} 2^{2}, 1^{4} 2^{2}, 2^{4}, 2^{4}, 4^{2}, 4^{2}$. Hence II $A$ shows dissimilar sets of partial indices for different heads. Therefore if a system $\Delta_{15}$ contains more than one $\Delta_{7}$, a determination of the partial indices of the seven triples of one head is not a sufficient characterization of the system. 
To determine if the six systems with fundamental Table II are distinct, we compare the indices of the thirty-five triples of each system, since systems with different sets of indices are incongruent. The indices for the 6 systems with fundamental seven-by-four array II are shown in the following table:

\begin{tabular}{|c|c|c|c|c|c|c|c|c|c|c|c|c|c|}
\hline $\mathrm{II}$ & $1^{12}$ & $1^{8} 2^{2}$ & $1^{6} 24$ & $1^{5} 2^{2}$ & $1^{4} 2^{4}$ & $1^{4} 26$ & $1^{4} 4^{2}$ & $1^{2} 2^{2} 5$ & $1^{2} 234$ & $1^{2} 2^{3} 4$ & $1^{2} 2^{2} 32$ & $1^{2} 46$ & 1236 \\
\hline$A$ & 1 & & 12 & & 10 & & & & & & 12 & & \\
$B$ & & & 2 & & 2 & 4 & 3 & 4 & 4 & 4 & 8 & & 4 \\
$C$ & & 2 & 1 & 6 & 5 & & 3 & 4 & 4 & & 4 & 2 & 4 \\
$D$ & & 2 & 1 & 6 & 4 & & 2 & 6 & 2 & 2 & 8 & 2 & \\
$E$ & 1 & 10 & 4 & 8 & 6 & & 2 & & 4 & 4 & 10 & & 4 \\
$F$ & & & 2 & & 5 & 4 & 2 & & 4 & 4 \\
\hline
\end{tabular}

This table shows 6 distinct systems differing from the 3 systems with fundamental seven-by-four array I.*

The sets of transitive elements for II $A$ are $a, b, c ; d, f, 2,4,5,8 ; e, g$, $1,3,6,7$; these with the classes separate the 35 triples of the system into 6 non-permutable su' 'divisions. The group is generated by $s_{1} \equiv(13)$ (24) (58) $(67), \quad s_{2} \equiv(a b)(d 2 f 4)(e 1 g 3)(58), \quad s_{3} \equiv(a b c)(d 48)(e 17)(f 25)$ $(g 36)$, and is of order 24 .

The sets of transitive elements for II $B$ are $a ; b, c ; d, f ; e ; g ; 1,3,6,7$; $2,4,5,8$; these with the classes separate the system into 13 non-permutable subdivisions. The group is generated by $s_{1} \equiv(b c)(1637)(2548)(d f)$, and is of order 4.

The sets of transitive elements for II $C$ are $a ; b ; c ; d ; e ; f ; g ; 1,3 ; 2,4 ; 5,8 ; 6,7$; these with the classes separate the system into 23 non-permutable subdivisions. The group is generated by $s_{1} \equiv(13)(24)(58)(67)$, and is of order 2.

The sets of transitive elements for II $D$ are $a ; b ; c ; d ; e ; f ; g ; 1,3 ; 2,4 ; 5,8$; 6,7 ; these with the classes separate the system into 23 non-permutable subdivisions. The group is generated by $s \equiv(13)(24)$ (58) (67), and is of order 2.

The two systems IIC and IID are distinct, since the number of triples appearing under each index is not the same for the two systems; but the group is identical for the two systems. This is another example of the fact that two distinct systems may have the same group.

The sets of transitive elements for III are $a ; b ; c ; d, f, 6,7 ; e, g, 5,8$; 1,$3 ; 2,4$; these with the classes separate the system into 12 non-permutable subdivisions. The group is generated by $s_{1} \equiv(13)(24)(58)(67)$, $s_{2} \equiv(d 7 f 6)(e 5 g 8)(13)(24)$, and is of order 8 .

\footnotetext{
* For the sake of brevity the work involved in the determination of the indices for every system is suppressed, and a brief outline of the process employed in obtaining the groups is given only for the system $I A$. A fuller elaboration is contained in a manuscript volume deposited in the Vassar College Library.
} 
The sets of transitive elements for II $F$ are $a ; b, c ; d ; f ; e, g ; 1,3,6,7$; $2,4,5,8$; these with the classes separate the system into 15 non-permutable subdivisions. The group is generated by $s_{1} \equiv(13)(24)(58)(67)$, $s_{2} \equiv(b c)(e g)(17)(28)(36)(45)$, and is of order 4 .

\section{SEVEN-BY-FOUR ARRAY WITH 14 QUADRANGULAR ARRAYS}

12. We construct next a seven-by-four array containing 14 quadrangular arrays, the greatest possible number, and we apply to this new array the re-heading process. The partial indices show 4 types of heads and we obtain the following systems:

\begin{tabular}{|c|c|c|c|c|c|c|c|c|c|c|}
\hline$a$ & $b$ & $c$ & $d$ & $e$ & $f$ & $g$ & \multirow[b]{2}{*}{ NUMBER } & & & \\
\hline $\begin{array}{l}12 \\
34 \\
56\end{array}$ & $\begin{array}{l}13 \\
24 \\
58\end{array}$ & $\begin{array}{l}14 \\
23 \\
57\end{array}$ & $\begin{array}{l}15 \\
26 \\
38\end{array}$ & $\begin{array}{l}16 \\
25 \\
37\end{array}$ & $\begin{array}{l}17 \\
28 \\
36\end{array}$ & $\begin{array}{l}18 \\
27 \\
35\end{array}$ & & $\begin{array}{l}\text { OF I } \\
\text { INDI }\end{array}$ & $\begin{array}{l}\text { RIPLES } \\
\text { CES }\end{array}$ & \\
\hline 78 & 67 & 68 & 47 & 48 & 45 & 46 & $1^{12}$ & $1^{8} 2^{2}$ & $1^{6} 3^{2}$ & $1^{4} 2^{4}$ \\
\hline $\begin{array}{l}a b c \\
a b c \\
a b c \\
a b d\end{array}$ & $\begin{array}{l}\text { ade } \\
\text { ade } \\
\text { adg } \\
\text { acg }\end{array}$ & $\begin{array}{l}\text { afg } \\
\text { afg } \\
\text { aef } \\
\text { aef }\end{array}$ & $\begin{array}{l}b d g \\
b d f \\
b d f \\
b c f\end{array}$ & $\begin{array}{l}\text { bef } \\
\text { beg } \\
\text { beg } \\
\text { beg }\end{array}$ & $\begin{array}{l}c d f \\
c d g \\
c d e \\
c d e\end{array}$ & $\begin{array}{l}c e g \\
c e f \\
c f g \\
d f g\end{array}$ & $\begin{array}{r}35 \\
7 \\
1\end{array}$ & $\begin{array}{l}24 \\
12\end{array}$ & $\begin{array}{l}16 \\
28\end{array}$ & $\begin{array}{l}4 \\
6 \\
7\end{array}$ \\
\hline
\end{tabular}

The system III $A$ contains one set of transitive elements $a, b, c, d, e, f, g$, $1,2,3,4,5,6,7,8$, and the thirty-five triples belong to one class.

The analysis of the Kirkman system by the method of sequences shows the index of each triple to be $1^{12}$, and the system III $A$ is congruent to the Kirkman system. The group for the system III $A$ is by far the most extensive of the 24 groups determined in this paper, and belongs to the earliest known $\Delta_{15}$.

The group for III $A$ is generated by $s_{1} \equiv(12)(34)(56)(78), s_{2} \equiv(13)$ (24) (58) (67), $s_{3} \equiv(15)(26)(38)(47), s_{4} \equiv(a c b)(e f g)(243)(678)$, $s_{5} \equiv(b c)(f g)(34)(78), \quad s_{6} \equiv(b c)(d f e g)(34)(5768), \quad s_{7} \equiv(a d g f c e b)$ $(1347286), s_{8} \equiv(a d 7 g f c 5368 b 1 e 42)$, and is of order $8 ! / 2 .^{*}$

The sets of transitive elements for III $B$ are $a ; b, c, d, e, f, g ; 1,2,3,4$, $5,6,7,8$. The group for the system is generated by $s_{1} \equiv(b e g)$ (cdf) (12) $(358467), s_{2} \equiv(d f)(e g)(57)(68), s_{3} \equiv(15)(26)(38)(47), s_{4} \equiv(d e)$ $(f g)(56)(78)$, and is of order 96 .

The sets of transitive elements for IIIC are $a, b, c ; d, e, f, g ; 1,2,3,4$, $5,6,7,8$. The group for the system IIIC is generated by $s_{1} \equiv(a b c)$ (dfg) (234) (578), $s_{2} \equiv(16)(25)(37)(48), s_{3} \equiv(12)(34)(56)(78)$, $s_{4} \equiv(d g)(e f)(58)(67)$, and is of order 96 . The groups for the systems III $B$ and III $C$ are of the same order 96 , but the sets of transitive elements for the two groups differ, hence the groups are distinct.

* C. Jordan (Traité des substitutions et des équations algébriques (1870), p. 380) states that the group for the Kirkman system is of order $8 ! / 2$, which agrees with the result obtained by the sequence method of analysis. 
The sets of transitive elements for IIID are $a, b, c, d, e, f, g ; 1,2,3,4$, $5,6,7,8$. The group is generated by $s_{1} \equiv(a c g)$ (bef) (248) (367), $s_{2} \equiv(15)(26)(38)(47), s_{3} \equiv$ (abedgcf) (3658472), $s_{4} \equiv(13)(24)(58)$ (67), and is of order 168 .

\section{SEVEN-BY-FOUR ARRAY WITH SIX QUADRANGUTAR ARRAYS}

13. We construct finally a seven-by-four array containing 6 quadrangular arrays, the only remaining number (cf. \$11) and we obtain the 4 following systems:

\begin{tabular}{|c|c|c|c|c|c|c|c|}
\hline & $a$ & $\boldsymbol{b}$ & $c$ & $d$ & $e$ & $f$ & $g$ \\
\hline & $\begin{array}{l}87 \\
65 \\
43 \\
21\end{array}$ & $\begin{array}{l}86 \\
75 \\
41 \\
23\end{array}$ & $\begin{array}{l}85 \\
76 \\
42 \\
31\end{array}$ & $\begin{array}{l}84 \\
73 \\
62 \\
15\end{array}$ & $\begin{array}{l}83 \\
74 \\
61 \\
25\end{array}$ & $\begin{array}{l}82 \\
71 \\
64 \\
53\end{array}$ & $\begin{array}{l}81 \\
72 \\
63 \\
54\end{array}$ \\
\hline & $\begin{array}{l}b d f \\
b d f \\
b d f \\
b d f\end{array}$ & $\begin{array}{l}a b c \\
a b e \\
a b e \\
a b c\end{array}$ & $\begin{array}{l}\text { beg } \\
\text { beg } \\
\text { cef } \\
\text { beg }\end{array}$ & $\begin{array}{l}\text { adg } \\
\text { adg } \\
\text { bcg } \\
\text { ade }\end{array}$ & $\begin{array}{l}\text { cde } \\
\text { cde } \\
\text { acd } \\
\text { cdg }\end{array}$ & $\begin{array}{l}\text { afe } \\
\text { acf } \\
\text { deg } \\
\text { afg }\end{array}$ & $\begin{array}{l}c f g \\
e f g \\
\text { afg } \\
e c f\end{array}$ \\
\hline
\end{tabular}

\begin{tabular}{|c|c|r|r|r|r|r|r|r|r|}
\hline IV & $1^{12}$ & $1^{8} 2^{2}$ & $1^{6} 24$ & $1^{6} 3^{2}$ & $1^{5} 2^{23}$ & $1^{4} 2^{4}$ & $1^{4} 26$ & $1^{4} 4^{2}$ & $1^{222} 3^{2}$ \\
\hline$A$ & 1 & 8 & & & 16 & 10 & & & \\
$B$ & & 1 & & 4 & 8 & 6 & 8 & 2 & 6 \\
$C$ & 1 & 10 & 4 & & 8 & 6 & & 2 & 4 \\
$D$ & 7 & 24 & & & & 4 & & & \\
\hline
\end{tabular}

The systems IVC and IVD show the same indices as the systems II $E$ and III $B$, respectively, and are congruent to them.

The sets of transitive elements for IV $A$ are $a, b ; c ; d, e, f, g, 1,2,3,4$; $5,6,7,8$; and the system contains 7 non-permutable subdivisions. The group for the system is generated by $s_{1} \equiv(a b)(d 1)(e 3)(f 2)(g 4)(67)$, $s_{2} \equiv(a b)(d 2 f 1)(e 4 g 3)(5687), \quad s_{3} \equiv(13)(24)(57)(68)$, and is of order 32.

The systems of transitive elements for IVB are $a ; b ; c ; d ; e ; f ; g ; 1,2,3,4$; $5,6,7,8$; and the system separates into 17 non-permutable subdivisions. The group is generated by $s_{1} \equiv(12)(34)(56)(78), s_{2} \equiv(14)(23)(58)$ (67), and is of order 4 .

Formation OF NEW SYSTEMS BY VARYING THE NUMBER OF PARTIAL QUADRANGULAR ARRAYS

14. The three sets of pairs 12,$34 ; 13,24 ; 14,23$ entering the seven-by-four array in 3 columns form a quadrangular array which has been denoted by the symbol [ $\left.{ }_{34}^{12}\right]$. Two of these sets of pairs occurring in the seven-by-four array in 2 columns form a partial quadrangular array, and may be denoted by the symbol $\left\{{ }_{34}^{12}\right\}$. A partial quadrangular array considered with respect to the two elements which head the columns in which the two sets of pairs enter, is designated in a recent paper as an "interlacing"* of the two ele-

* F. N. Cole, The triad systems of thirleen elements. These Trans a c ti o n s, vol. 14 (1913), pp. 1-5. 
ments which head the columns. Non-congruent triple-systems may be formed by keeping constant the number of quadrangular arrays in the sevenby-four array, and varying the number of partial quadrangular arrays.

The seven-by-four array II contains two quadrangular arrays $\left[\begin{array}{c}12 \\ 34\end{array}\right],\left[\begin{array}{c}56 \\ 78\end{array}\right]$ and eight partial quadrangular arrays $\left\{\begin{array}{c}12 \\ 68\end{array}\right\},\left\{\begin{array}{c}12 \\ 78\end{array}\right\},\left\{\begin{array}{l}18 \\ 68\end{array}\right\},\left\{\begin{array}{c}13 \\ 57\end{array}\right\},\left\{\begin{array}{c}24 \\ 58\end{array}\right\},\left\{\begin{array}{c}24 \\ 67\end{array}\right\},\left\{\begin{array}{c}34 \\ 68\end{array}\right\}$, $\left\{{ }_{78}^{34}\right\}$. The seven-by-four array $V$, given below, contains the same number of quadrangular arrays, $\left[\begin{array}{c}12 \\ 58\end{array}\right],\left[\begin{array}{l}34 \\ 67\end{array}\right]$, but a different number of partial quadrangular arrays, the twelve $\left\{\begin{array}{l}12 \\ 16\end{array}\right\},\left\{\begin{array}{l}12 \\ 37\end{array}\right\},\left\{\begin{array}{c}13 \\ 48\end{array}\right\},\left\{\begin{array}{c}13 \\ 58\end{array}\right\},\left\{\begin{array}{c}14 \\ 57\end{array}\right\},\left\{\begin{array}{c}16 \\ 78\end{array}\right\},\left\{\begin{array}{c}28 \\ 45\end{array}\right\},\left\{\begin{array}{c}23 \\ 68\end{array}\right\},\left\{\begin{array}{c}24 \\ 78\end{array}\right\},\left\{\begin{array}{c}25 \\ 67\end{array}\right\}$, $\left\{\begin{array}{l}35 \\ 78\end{array}\right\},\left\{\begin{array}{l}45 \\ 68\end{array}\right\}$. The re-heading process applied to this seven-by-four array shows six types of heads.

\begin{tabular}{|c|c|c|c|c|c|c|c|c|c|c|c|}
\hline \multirow{3}{*}{$\mathbf{v}$} & \multirow{3}{*}{$\begin{array}{c}a \\
12 \\
58 \\
34 \\
67\end{array}$} & \multirow{3}{*}{$\begin{array}{c}b \\
15 \\
28 \\
37 \\
46\end{array}$} & \multirow{3}{*}{$\begin{array}{c}c \\
18 \\
25 \\
36 \\
47\end{array}$} & \multirow{3}{*}{$\begin{array}{l}14 \\
26 \\
38 \\
57\end{array}$} & \multirow{3}{*}{$\begin{array}{c}e \\
17 \\
23 \\
45 \\
68\end{array}$} & \multirow{3}{*}{$\begin{array}{c}f \\
13 \\
27 \\
48 \\
56\end{array}$} & \multirow{3}{*}{$\begin{array}{l}16 \\
24 \\
35 \\
78\end{array}$} & \multirow{2}{*}{\multicolumn{4}{|c|}{$\begin{array}{l}\text { NUMBER OF TRIPLES WITH } \\
\text { PARTIAL INDICES }\end{array}$}} \\
\hline & & & & & & & & & & & \\
\hline & & & & & & & & $\mathbf{1}^{8}$ & $1^{4} 2^{2}$ & 24 & $\mathbf{4}^{2}$ \\
\hline $\begin{array}{l}\boldsymbol{A} \\
\boldsymbol{B} \\
\boldsymbol{C} \\
\boldsymbol{D} \\
\boldsymbol{E} \\
\boldsymbol{F}\end{array}$ & $\begin{array}{l}a b c \\
a b d \\
a b d \\
a b d \\
a b c \\
a b c\end{array}$ & $\begin{array}{l}\text { ade } \\
\text { ace } \\
\text { ace } \\
\text { acf } \\
\text { ade } \\
\text { adf }\end{array}$ & $\begin{array}{l}\text { afg } \\
\text { afg } \\
\text { aff } \\
\text { aeg } \\
\text { afg } \\
\text { aeg }\end{array}$ & $\begin{array}{l}b d f \\
b c f \\
b c g \\
b c g \\
b d g \\
b d g\end{array}$ & $\begin{array}{l}\text { beg } \\
\text { beg } \\
\text { bef } \\
\text { bef } \\
\text { bef } \\
\text { bef }\end{array}$ & $\begin{array}{l}\text { cdg } \\
\text { cdg } \\
\text { cdf } \\
\text { cde } \\
\text { cdf } \\
\text { cde }\end{array}$ & $\begin{array}{l}\text { cef } \\
\text { def } \\
\text { deg } \\
\text { dfg } \\
\text { ceg } \\
\text { cfg }\end{array}$ & $\begin{array}{l}1 \\
1\end{array}$ & $\begin{array}{l}2 \\
3 \\
4 \\
6\end{array}$ & $\begin{array}{l}6 \\
4 \\
2 \\
1 \\
2\end{array}$ & $\begin{array}{l}\mathbf{3} \\
\mathbf{3} \\
\mathbf{3}\end{array}$ \\
\hline
\end{tabular}

A determination of the indices of the 6 systems with fundamental seven-byfour array $\mathrm{V}$ gives the following result:

\begin{tabular}{|c|c|c|c|c|c|c|c|c|c|c|}
\hline $\mathrm{V}$ & $1^{12}$ & $1^{8} 2^{2}$ & $1^{6} 3^{2}$ & $1^{6} 2^{2} 3$ & $1^{4} 2^{4}$ & $1^{4} 35$ & $1^{4} 4^{2}$ & $1^{3} 234$ & $1^{3} 3^{3}$ & $1^{2} 2^{2} 3^{2}$ \\
\hline$A$ & 1 & & & & 18 & & & & 16 & \\
$B$ & & & & & 4 & & 3 & 12 & 4 & 12 \\
$C$ & & 2 & & 4 & 6 & 8 & 3 & 4 & & 8 \\
$D$ & & 3 & 4 & 16 & 7 & 12 & 3 & & & 6 \\
$E$ & 1 & 8 & 16 & 16 & 6 & & & & & \\
$F$ & 1 & 12 & 16 & & 6 & & & & & \\
\hline
\end{tabular}

The triple-systems VE and VF show the same sets of indices as IV $A$ and IIIC, respectively, and are congruent to them. The analysis follows for the 4 systems $\mathrm{V} A, \mathrm{~V} B, \mathrm{~V} C, \mathrm{~V} D$, which are distinct from the preceding 15 triplesystems.

The sets of transitive elements for $\mathrm{V} A$ are $a, b, c ; d, e, f, g, 1,2,3,4,5$, $6,7,8$. The group is generated by $s_{1} \equiv(a b c)(e f g)(258)(367), s_{2} \equiv(b c)$ $(d 1)(e 2)(f 8)(g 5)(67), s_{3} \equiv(d 14)(e 23)(f 56)(g 87), s_{4} \equiv(a c b)(d f g)$ (182) (376), and is of order 288.

The sets of transitive elements for $\mathrm{V} B$ are $a, b, c ; d, e, f ; g ; 1,2,3,4,5$, $6,7,8$; these with the classes separate the system into 6 non-permutable subdivisions. The group is generated by $s_{1} \equiv(a b)(e f)(1327)(4865)$, $s_{2} \equiv(a b)(e f)(14)(26)(35)(78), s_{3} \equiv(a c b)(d e f)(182)(467)$, and is of order 24 . 
The sets of transitive elements for $\mathrm{V} C$ are $a ; b, c ; d, e ; f ; g ; 1,2,3,4,5$, $6,7,8$; these with the classes separate the system into 11 non-permutable subdivisions. The group for the system is generated by $s_{1} \equiv(b c)$ (de) (16) (27) (35) (48), $s_{2} \equiv(b c)(d e)(1457)(2386)$, and is of order 8 .

The sets of transitive elements for VD are $a, b, c ; d, f, g ; e ; 1,2,5,8$; $3,4,6,7$; these with the classes separate the system into 7 non-permutable subdivisions. The group for the system is generated by $s_{1} \equiv(12)$ (37) (46) $(58), s_{2} \equiv(18)(25)(34)(67), s_{3} \equiv(a c b)(d f g)(125)(347)$, and is of order 12 .

\section{ANALYSIS OF TRIPLE-SYSTEMS FORMED BY OTHER METHODS}

15. A few of the well-known systems $\Delta_{15}$ are now examined, in order to determine, if possible, systems incongruent to the 19 already given.

(i) Analysis of the Carpmael systems. - The indices for the systems given by Carpmael* show two non-congruent systems. One of these yields a set of indices identical with that of $\mathrm{V} A$; hence this system is omitted from further examination. The other Carpmael system yields a new set of indices; therefore we determine in this system a $\Delta_{7}$, arrange the system in the form of a seven-by-four array, and apply the re-heading process, thus obtaining four systems incongruent to the preceding 19 , VI $A, \operatorname{VI} B, \operatorname{VI} C$, and VID of the following table:

\begin{tabular}{|c|c|c|c|c|c|c|c|c|c|c|c|c|c|c|}
\hline & $a$ & $\boldsymbol{b}$ & $c$ & $d$ & $e$ & $f$ & $g$ & & & & & & & \\
\hline $\begin{array}{l}\text { VIA } \\
\text { VIB } \\
\text { VIC } \\
\text { VID }\end{array}$ & $\begin{array}{l}12 \\
34 \\
58 \\
67\end{array}$ & $\begin{array}{l}17 \\
23 \\
48 \\
56\end{array}$ & $\begin{array}{l}16 \\
24 \\
38 \\
57\end{array}$ & $\begin{array}{l}15 \\
28 \\
36 \\
47\end{array}$ & $\begin{array}{l}18 \\
27 \\
35 \\
46\end{array}$ & $\begin{array}{l}14 \\
25 \\
37 \\
68\end{array}$ & $\begin{array}{l}13 \\
26 \\
45 \\
78\end{array}$ & $\begin{array}{l}a b e \\
a b c \\
a b d \\
a b g\end{array}$ & $\begin{array}{l}\text { acf } \\
\text { adg } \\
\text { acg } \\
\text { ace }\end{array}$ & $\begin{array}{l}\text { adg } \\
\text { aef } \\
\text { aef } \\
\text { adf }\end{array}$ & $\begin{array}{l}\text { bdf } \\
\text { bde } \\
\text { bce } \\
\text { bed }\end{array}$ & $\begin{array}{l}\text { cde } \\
\text { bfg } \\
\text { bfg } \\
\text { bef }\end{array}$ & $\begin{array}{l}e f g \\
c d f \\
c d f \\
c f g\end{array}$ & $\begin{array}{l}\text { beg } \\
\text { ceg } \\
\text { deg } \\
\text { deg }\end{array}$ \\
\hline
\end{tabular}

A comparison of the two seven-by-four arrays I and VI shows that neither contains any quadrangular array, but that while I contains no partial quad-

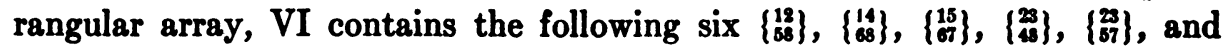
$\left\{\begin{array}{l}33 \\ 3\end{array}\right\}$. Hence the two sets of systems I and VI may be formed by constructing seven-by-four arrays, which contain a fixed number (zero) of quadrangular arrays and a variable number of partial quadrangular arrays. The analysis and the groups for the system VI are given below.

\begin{tabular}{|c|c|c|c|c|c|c|c|c|c|c|c|c|c|c|c|c|c|}
\hline & 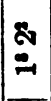 & $\mid \begin{array}{l}\mathbf{N} \\
\vdots \\
\vdots\end{array}$ & 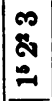 & $\underline{\Delta}$ & $\begin{array}{l}\text { Z } \\
\vdots \\
-1\end{array}$ & 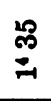 & 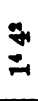 & $\begin{array}{l}\text { م } \\
\text { సี } \\
\text { జ }\end{array}$ & $\begin{array}{l}\text { 苛 } \\
\text { 口 }\end{array}$ & 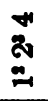 & 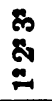 & is & 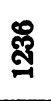 & స్తి & జ0 & $\begin{array}{l}\infty \\
\text { సె }\end{array}$ & ส \\
\hline $\begin{array}{l}\text { VIA } \\
\text { VIB } \\
\text { VIC } \\
\text { VID }\end{array}$ & $\begin{array}{l}1 \\
3\end{array}$ & $\begin{array}{l}\mathbf{2} \\
\mathbf{3} \\
\mathbf{3}\end{array}$ & 6 & $\begin{array}{l}\mathbf{4} \\
2 \\
1 \\
1\end{array}$ & $\begin{array}{l}3 \\
1\end{array}$ & $\begin{array}{l}2 \\
3\end{array}$ & $\begin{array}{l}1 \\
3\end{array}$ & $\begin{array}{l}\mathbf{6} \\
\mathbf{3}\end{array}$ & 2 & $\begin{array}{l}\mathbf{2} \\
\mathbf{3} \\
\mathbf{3}\end{array}$ & $\begin{array}{l}8 \\
6 \\
6\end{array}$ & $\begin{array}{l}2 \\
9 \\
6\end{array}$ & $\begin{array}{r}12 \\
2 \\
6\end{array}$ & $\begin{array}{l}4 \\
3\end{array}$ & $\begin{array}{l}6 \\
1\end{array}$ & 2 & $\begin{array}{l}4 \\
1 \\
1\end{array}$ \\
\hline
\end{tabular}

- Some solutions of Kirkman's 15-school-girl problem, P r o c e e d ing a of th e L o n d o n Mathematical S o ciety, vol. 12 (1881), pp. 148-156. 
The sets of transitive elements for VIA are $a, b, c, d, e, f ; g ; 1,2,4$, $7 ; 3,5,6,8$. The group is generated by $s_{1} \equiv(a b e)(c f d)(172)(386)$, $s_{2} \equiv(a d)(b c)(14)(27)(35)(68), s_{3} \equiv(b c)(e f)(12)(36)(47)(58)$, and is of order 12.

The sets of transitive elements for VIB are $a ; b, c ; d ; e, f ; g ; 1,2 ; 3,6$; 4,$7 ; 5,8$. These with the classes separate the system into 21 non-permutable subdivisions. The group is of order 2 and is generated by $8 \equiv(b c)$ (ef) (12) (36) (47) (58).

The sets of transitive elements for VIC are $a, b, e ; c, d, f ; g ; 1,2,7 ; 4 ; 5$; $3,6,8$. These with the classes separate the system into 13 non-permutable subdivisions. The group for the system is generated by $s \equiv(a e b)$ (cdf) (127) (368), and is of order 3.

The sets of transitive elements for VID are $a, c, e ; b, d, f ; g ; 1,4,7 ; 2 ; 6$; $3,5,8$. These with the classes separate the system into 13 non-permutable subdivisions. The group for the system is generated by $s \equiv(a c e)(b f d)$ (147) (358), and is of order 3.

A comparison of the systems IB, IC, VIC, VID, shows different sets of indices but identical groups. Here then are four incongruent systems with the same group (cf. $\$ 8$ and \$9). Therefore it is evident that the identity of the groups, though a necessary condition for the congruency of two triple-systems, is not sufficient.

(ii) Analysis of the Heffter systems. - The Heffter* method of constructing triple-systems of $6 m+3$ elements furnishes two systems for $6 m+3=15$. The first of these contains 15 systems $\Delta_{7}$, the index of each triple is $1^{12}$, and the system shows the same set of indices as the system here denoted by III $A$. The second system contains no $\Delta_{7}$, and is therefore distinct from the preceding 23 systems, each of which contains at least one $\Delta_{7}$. This second system is now denoted by VII. Its analysis is shown in the following table:

Classification of VII by indices

\begin{tabular}{|c|c|c|c|c|c|c|c|c|c|c|c|}
\hline \multicolumn{2}{|c|}{4,8} & \multicolumn{10}{|c|}{3,9} \\
\hline $\begin{array}{l}\text { aft } \\
\text { bg5 } \\
c 16\end{array}$ & $\begin{array}{l}d 27 \\
e 38\end{array}$ & $\begin{array}{l}a 58 \\
\text { ade } \\
a b 6\end{array}$ & $\begin{array}{l}a g 7 \\
a c 2 \\
a 13\end{array}$ & $\begin{array}{l}\text { bc7 } \\
\text { bef } \\
b 18\end{array}$ & $\begin{array}{l}b 24 \\
c d 8 \\
c f g\end{array}$ & $\begin{array}{l}c e 4 \\
c 35 \\
d g 1\end{array}$ & $\begin{array}{l}d f 5 \\
d 46 \\
e 12\end{array}$ & $\begin{array}{l}e g 6 \\
e 57 \\
\text { f23 }\end{array}$ & $\begin{array}{l}f 17 \\
f 68 \\
g 34\end{array}$ & $\begin{array}{l}g 28 \\
145 \\
256\end{array}$ & $\begin{array}{l}367 \\
478 \\
b d 3\end{array}$ \\
\hline
\end{tabular}

The system contains 1 set of transitive elements: $a, b, c, d, e, f, g, 1,2,3$, $4,5,6,7,8$, and separates into 2 non-permutable subdivisions. The group is generated by $s_{1} \equiv(a d 63)(c 8 f 2)(e 471), s_{2} \equiv(a b c d e f g 12345678)$, and is of order 60 .

(iii) Netto $\dagger$ describes two methods for constructing triple-systems of 15

* L. Heffter, Ueber Tripelsysteme, Mathemat is che An a le n, vol. 49 (1897), pp. 101-112.

† L. c. 
elements. The analysis of the two systems by the method of sequences shows that the index of every triple in each system is $1^{12}$ and the two systems are of the type here designated by III $A$.

(iv) E. H. Moore* gives different methods for constructing triple-systems. A system formed by one of these methods was found to be of the type IIIA. Under the re-heading process a system formed by a second method yielded 4 systems congruent, respectively, to the systems here designated as IIE, III $B, \operatorname{III} C, \operatorname{IV} A$.

16. We have shown for the two fundamental seven-by-four arrays containing, respectively, zero and two quadrangular arrays, that non-congruent systems may be formed by varying the number of partial quadrangular arrays. It seems possible that additional systems $\Delta_{15}$, each containing at least one $\Delta_{7}$, might be formed by the same method from these two arrays, and also from the two fundamental arrays containing, respectively, six and fourteen quadrangular arrays. The purpose of this investigation is primarily to exhibit a new method of comparison for triple-systems, and while several systems $\Delta_{15}$ not hitherto constructed have been obtained, no exhaustive determination of all possible systems $\Delta_{15}$ has been undertaken.

The method of comparison here investigated is applicable to any system $\Delta_{2 t+1}$ by merely arranging the system in the form of a $2 t+1-b y-t$ array and determining the indices for this array. In constructing systems $\Delta_{15}$ which contain no $\Delta_{7}$, the number of partial quadrangular arrays adinitted into a fifteen-by-seven array must be considered, but for systems $\Delta_{15}$ which contain one or more systems $\Delta_{7}$, the number of complete and partial quadrangular arrays admitted into a seven-by-four array is to be considered. Exactly similar conditions exist in the case of systems $\Delta_{2 t+1}$. In constructing systems $\Delta_{2 t+1}$ which contain no $\Delta_{t}$, the consideration of a $2 t+1$-by- $t$ array is necessary, but for systems $\Delta_{2 t+1}$ which contain one or more systems $\Delta_{t}$, the consideration of a $t$-by- $-\frac{1}{2}(t+1)$ array is sufficient.

April, 1913

* L. c. 